COMMENTARY

\title{
Caring for Meningiomas: A Multi-Disciplinary Approach
}

\section{Uri P Hadelsberg, $M D^{*}$ and Nevo Margalit, $M D$}

Department of Neurosurgery, Shaare Zedek Medical Center, Jerusalem, Israel

*Corresponding author: Uri P Hadelsberg, MD, Department of Neurosurgery, Shaare Zedek Medical Center, 9103102, Jerusalem, Israel

The authors hereby present a case managed by a multi-disciplinary team involving a huge intracranial meningioma. This patient is a 36-year-old female which presented to our institution with headaches, gait disturbances, visual disturbances and dizziness which began 3 months prior to her admission (Figure 1). Her medical history is noticeable for leukemia at age 6 which was

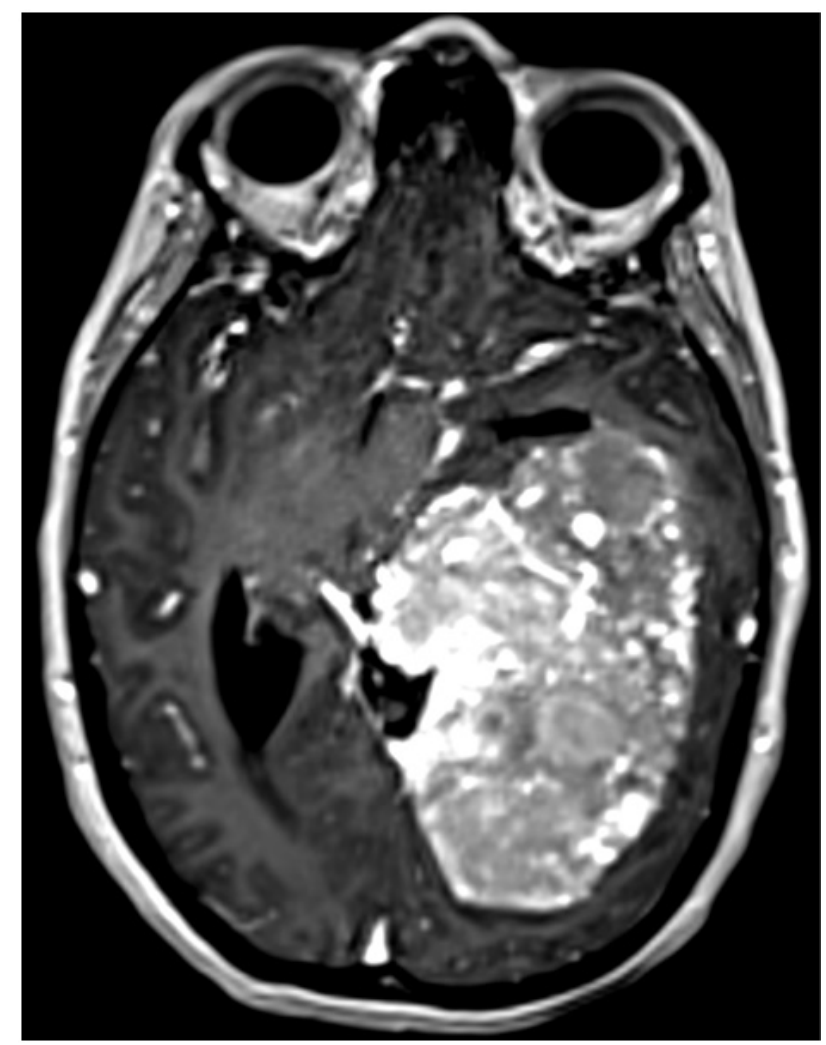

Figure 1: A gigantic left supra and infra-tentorial meningioma in a 36-year-old female. Notice the blood vessels supplying the tumor. treated with chemotherapy and radiation to the head, with no current active malignancy. Upon admission the patient underwent a CT scan and a subsequent MRI which revealed a highly vascularized, gigantic $9.3 \times 6.1$ $\mathrm{cm}$ left supra and infra-tentorial brain lesion suspected for a meningioma causing severe mass effect.

This patient was admitted to our department and eventually underwent surgery with gross total resection after pre-operative endovascular embolization of the tumor. The patient underwent a successful recovery and was discharged without major neurological deficits to rehab. On follow up the patient is doing well and is back home with her children.

On the day of surgery the patient was intubated and underwent cerebral embolization with occlusion of several tumor-feeding arteries. The patient was then transferred to the operating room while intubated and underwent craniotomy and gross total tumor resection.

In our department each surgical candidate's tumor is brought to discussion prior to surgery. We discuss the patient, his/her medical status, should he be operated on, and what we plan on achieving during surgery. We also debate the different surgical approaches and whether-or-not any other treatment is required as was in this patient. It is important to be honest about treatment goals as well as be modest regarding outcomes for each treatment protocol. The treatment armamentarium for patients with brain tumors has evolved to so many different specialties and potential therapeutic agents that one may be startled and a bit overwhelmed from the options (Figure 2).

A serious, thorough debate in our department not only provides us with knowledge for the treatment of

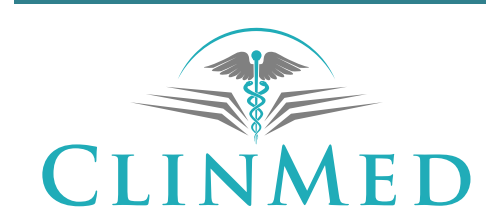

INTERNATIONAL LIBRARY
Citation: Hadelsberg UP, Margalit N (2019) Caring for Meningiomas: A Multi-Disciplinary Approach. Neurosurg Cases Rev 2:020. doi.org/10.23937/2643-4474/1710020

Accepted: June 27, 2019; Published: June 29, 2019

Copyright: (C) 2019 Hadelsberg UP, et al. This is an open-access article distributed under the terms of the Creative Commons Attribution License, which permits unrestricted use, distribution, and reproduction in any medium, provided the original author and source are credited. 


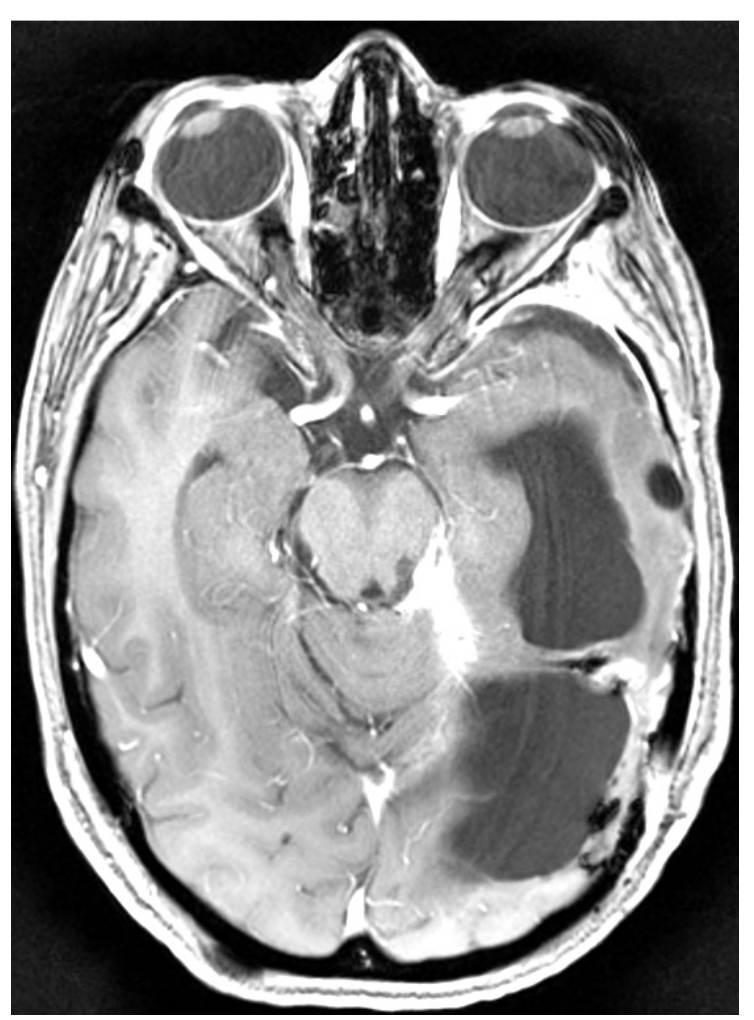

Figure 2: 8 months post-treatment, in a routine follow up of the patient in Figure 1. Notice gross total resection and improvement in the amount of brain shifting which was noticed prior to surgery.

future patients but also provides us with a clearer picture to what pathology the patient has and what are the best treatment options for him/her. One of the important pre-operative requirements when dealing with menigiomas this big is organizing the logistics and executing a smooth operative course with the least amount of disruptions possible. This necessitates excellent communication between caretakers.

Prior to surgery both the neurosurgeon, neuroradiologist, interventional radiologist (IR) as well as the anesthesiologist met together and planned the oper- ative course during the day. A stepwise approach was planned which included minute details such as patient transfer from the IR suite to the operative theater as well as discussing surgical and interventional goals. Having both the neuroradiologist, neurosurgeon and IR physician go through the imaging helped in delineating which vessels can and should be occluded and detailing the pitfalls during surgery. We believe that in debating such a case and thoroughly pre-planning treatment in an honest and healthy manner makes us more alert to problems which may occur during surgery and how to avoid them. After the necessary steps were planned, a meeting with the patient and her family was scheduled. During the meeting both treating physicians explained the risks of surgery and IR intervention as well as any neurological deterioration expected.

Cerebral embolization is not a new modality, yet its deployment in the setting of gigantic meningiomas is yet to be standard practice. Our initial treatment modality of embolization managed to close the main blood supply of the brain tumor, which consequently lead to a substantial decrease in blood loss during surgery and what we believe is less surgical maneuvers needed in order to debulk the tumor.

The learning lesson in this case is raising awareness to the amount of cooperation and dialogue between treating physicians which is needed in order to achieve a successful treatment and satisfying results in such complex patients.

To summarize, these treatment strategies should be employed for every patient prior to surgery, minimalizing his/her chances of a bad outcome. A relevant and honest discussion between physicians should be done. A discussion which involves a multi-disciplinary board of treating physicians as needed, something which will ensure these bad outcomes stay out of harm's way.

The authors report no conflict of interest. 